Theoretical Study of the Pyrolysis of Methyltrichlorosilane in the Gas Phase.

\title{
I: Thermodynamics
}

\section{Supplementary Materials}

A. Energies (Hartree) and zero point energies (Hartree) of the studied species.

\begin{tabular}{|c|c|c|c|c|c|}
\hline \multirow{2}{*}{ Species } & \multicolumn{2}{|c|}{ MP2 } & \multicolumn{2}{|c|}{$\operatorname{CCSD}(\mathrm{T})$} & \multirow{2}{*}{$\mathrm{ZPE}^{\mathrm{b}}$} \\
\hline & Aug-cc-pVDZ & Aug-cc-pVTZ & Aug-cc-pVDZ & Aug-cc-pVTZ & \\
\hline 1. $\mathrm{Cl}$ & -459.592150 & -459.647332 & -459.612222 & -459.667404 & 0.000000 \\
\hline 2. $\mathrm{Cl}_{2}$ & -919.259803 & -919.386486 & -919.295152 & -919.421835 & 0.001233 \\
\hline 3. $\mathrm{H}$ & -0.499334 & -0.499821 & -0.499334 & -0.499821 & 0.000000 \\
\hline 4. $\mathrm{H}_{2}$ & -1.156216 & -1.164818 & -1.164870 & -1.173472 & 0.010086 \\
\hline 5. $\mathrm{HCl}$ & -460.251823 & -460.315026 & -460.272292 & -460.335495 & 0.006934 \\
\hline 6. $\mathrm{C}_{2} \mathrm{H}$ & -76.361059 & -76.424608 & -76.405614 & -76.469163 & 0.017135 \\
\hline 7. $\mathrm{C}_{2} \mathrm{H}_{2}$ & -77.092997 & -77.163163 & -77.121362 & -77.191528 & 0.025199 \\
\hline 8. $\mathrm{C}_{2} \mathrm{H}_{3}$ & -77.640365 & -77.710135 & -77.686962 & -77.756732 & 0.038298 \\
\hline 9. $\mathrm{C}_{2} \mathrm{H}_{3} \mathrm{Cl}$ & -537.394050 & -537.526833 & -537.444840 & -537.577623 & 0.042721 \\
\hline 10. $\mathrm{C}_{2} \mathrm{H}_{4}$ & -78.328918 & -78.403863 & -78.369593 & -78.444538 & 0.051091 \\
\hline 11. $\mathrm{C}_{2} \mathrm{H}_{5}$ & -78.885759 & -78.963392 & -78.931381 & -79.009014 & 0.059863 \\
\hline 12. $\mathrm{C}_{2} \mathrm{H}_{5} \mathrm{Cl}$ & -538.616889 & -538.757255 & -538.674541 & -538.814907 & 0.066852 \\
\hline 13. $\mathrm{C}_{2} \mathrm{H}_{6}(\mathrm{e})$ & -79.546313 & -79.630158 & -79.593247 & -79.677092 & 0.074365 \\
\hline 14. $\mathrm{C}_{2} \mathrm{H}_{6}$ & -79.551122 & -79.634782 & -79.597877 & -79.681537 & 0.074879 \\
\hline 15. ${ }^{1} \mathrm{CH}_{2}$ & -38.999960 & -39.034168 & -39.031507 & -39.065715 & 0.016901 \\
\hline 16. ${ }^{3} \mathrm{CH}_{2}$ & -39.025270 & -39.057318 & -39.048293 & -39.080341 & 0.017576 \\
\hline 17. $\mathrm{CH}_{2} \mathrm{C}$ & -77.016377 & -77.080725 & -77.058296 & -77.122644 & 0.023494 \\
\hline 18. $\mathrm{CH}_{2} \mathrm{Cl}$ & -498.761089 & -498.859088 & -498.799853 & -498.897852 & 0.023202 \\
\hline 19. $\mathrm{CH}_{2} \mathrm{Cl}_{2}$ & -958.483982 & -958.644651 & -958.534008 & -958.694677 & 0.029596 \\
\hline 20. $\mathrm{CH}_{3}$ & -39.698266 & -39.737984 & -39.724652 & -39.764370 & 0.030009 \\
\hline 21. $\mathrm{CH}_{3} \mathrm{CH}(\mathrm{s})$ & -78.201127 & -78.274142 & -78.250309 & -78.323324 & 0.046484 \\
\hline 22. $\mathrm{CH}_{3} \mathrm{Cl}$ & -499.425314 & -499.528855 & -499.464795 & -499.568336 & 0.037899 \\
\hline 23. $\mathrm{CH}_{4}$ & -40.367727 & -40.414099 & -40.395770 & -40.442142 & 0.044978 \\
\hline 24. $\mathrm{HCHC}$ & -77.018798 & -77.085560 & -77.052831 & -77.119593 & 0.022567 \\
\hline 25. $\mathrm{Si}_{2} \mathrm{Cl}_{4}$ & -2416.795382 & -2417.100961 & -2416.884234 & -2417.189813 & 0.007445 \\
\hline 26. $\mathrm{Si}_{2} \mathrm{Cl}_{5}$ & -2876.523769 & -2876.895898 & -2876.619852 & -2876.991981 & 0.009889 \\
\hline 27. $\mathrm{Si}_{2} \mathrm{Cl}_{6}$ & -3336.280123 & -3336.720399 & -3336.386989 & -3336.827265 & 0.012022 \\
\hline 28. $\mathrm{SiCl}_{2}$ & -1208.387517 & -1208.536946 & -1208.434219 & -1208.583648 & 0.002711 \\
\hline 29. $\mathrm{SiCl}_{3}$ & -1668.080101 & -1668.296437 & -1668.135308 & -1668.351644 & 0.004936 \\
\hline 30. $\mathrm{SiCl}_{4}$ & -2127.836605 & -2128.122765 & -2127.903246 & -2128.189406 & 0.007186 \\
\hline 31. $\mathrm{SiH}_{2} \mathrm{Cl}$ & -749.841901 & -749.936509 & -749.880564 & -749.975172 & 0.016761 \\
\hline
\end{tabular}




\begin{tabular}{|c|c|c|c|c|c|}
\hline 32. $\mathrm{SiH}_{2} \mathrm{Cl}_{2}$ & -1209.602226 & -1209.764787 & -1209.653189 & -1209.815750 & 0.020608 \\
\hline 33. $\mathrm{SiH}_{3} \mathrm{Cl}$ & -750.482807 & -750.584425 & -750.525653 & -750.627271 & 0.026436 \\
\hline 34. $\mathrm{SiHCl}$ & -749.247058 & -749.336515 & -749.286667 & -749.376124 & 0.007759 \\
\hline 35. $\mathrm{SiHCl}_{2}$ & -1208.961443 & -1209.116672 & -1209.008490 & -1209.163719 & 0.011188 \\
\hline 36. $\mathrm{SiHCl}_{3}$ & -1668.720895 & -1668.945075 & -1668.779828 & -1669.004008 & 0.014114 \\
\hline 37. $\mathrm{CH}_{2} \mathrm{SiCl}_{2}$ & -1247.547685 & -1247.740684 & -1247.608112 & -1247.801111 & 0.028191 \\
\hline 38. $\mathrm{CH}_{2} \mathrm{SiCl}_{3}$ & -1707.262019 & -1707.519594 & -1707.337442 & -1707.595017 & 0.028822 \\
\hline 39. $\mathrm{CH}_{2} \mathrm{SiHCl}$ & -788.433248 & -788.565407 & -788.486028 & -788.618187 & 0.034340 \\
\hline 40. $\mathrm{CH}_{2} \mathrm{SiHCl}_{2}$ & -1248.142084 & -1248.338473 & -1248.209642 & -1248.406031 & 0.035608 \\
\hline 41. $\mathrm{CH}_{3} \mathrm{SiCl}$ & -788.452491 & -788.582723 & -788.508582 & -788.638814 & 0.036803 \\
\hline 42. $\mathrm{CH}_{3} \mathrm{SiCl}_{2}$ & -1248.167709 & -1248.362922 & -1248.231734 & -1248.426947 & 0.040046 \\
\hline 43. $\mathrm{CH}_{3} \mathrm{SiCl}_{2} \mathrm{Cl}$ & -1707.740825 & -1707.995554 & -1707.836854 & -1708.091583 & 0.041553 \\
\hline 44. $\mathrm{CH}_{3} \mathrm{SiCl}_{3}$ & -1707.930906 & -1708.194049 & -1708.006580 & -1708.269723 & 0.042603 \\
\hline 45. $\mathrm{CH}_{3} \mathrm{SiH}_{2} \mathrm{Cl}$ & -789.686496 & -789.828561 & -789.746489 & -789.888554 & 0.055501 \\
\hline 46. $\mathrm{CH}_{3} \mathrm{SiHCl}$ & -789.044361 & -789.179508 & -789.100181 & -789.235328 & 0.046112 \\
\hline 47. $\mathrm{CH}_{3} \mathrm{SiHCl}_{2}$ & -1248.809614 & -1249.011975 & -1248.877533 & -1249.079894 & 0.049239 \\
\hline 48. $\mathrm{CHSiCl}$ & -787.149935 & -787.271738 & -787.198463 & -787.320266 & 0.014522 \\
\hline 49. ${ }^{1} \mathrm{CHSiCl}_{3}$ & -1706.560802 & -1706.814749 & -1706.637411 & -1706.891358 & 0.018334 \\
\hline $50 .{ }^{3} \mathrm{CHSiCl}_{3}$ & -1706.593501 & -1706.844976 & -1706.667157 & -1706.918632 & 0.017021 \\
\hline
\end{tabular}

a. Estimated CCSD(T)/aug-cc-pVTZ energies given by Equation 1 in the paper.

b. Zero point energies (ZPE) are calculated at the MP2/aug-cc-pVDZ level. 
B. Cartesian coordinates (angstrom) at the MP2/aug-cc-pVDZ level.

Species 1.

Cl $\quad .0000000000 \quad .0000000000 \quad .0000000000$

Species 2.

$\begin{array}{llll}\mathrm{Cl} & .0000000000 & .0000000000 & 1.0194970074\end{array}$

$\begin{array}{lllll}\mathrm{Cl} & .0000000000 & .0000000000 & -1.0194970074\end{array}$

Species 3.

$\begin{array}{llll}\mathrm{H} & .0000000000 & .0000000000 & .0000000000\end{array}$

Species 4.

$\begin{array}{llll}\mathrm{H} & .0000000000 & .0000000000 & -.3774655572 \\ \mathrm{H} & .0000000000 & .0000000000 & .3774655572\end{array}$

$\begin{array}{llll}\mathrm{H} & .0000000000 & .0000000000 & .3774655572\end{array}$

Species 5.

$\mathrm{H} \quad .0000000000 \quad .0000000000 \quad-.0089725260$

$\begin{array}{lllll}\mathrm{Cl} & .0000000000 & .0000000000 & 1.2789725260\end{array}$

Species 6.

$\begin{array}{llll}\mathrm{C} & .0000000000 & .0000000000 & -.6023463089\end{array}$

$\begin{array}{llll}\mathrm{C} & .0000000000 & .0000000000 & .5888747269\end{array}$

$\mathrm{H} \quad \quad .0000000000 \quad .0000000000 \quad-1.6763420500$

Species 7.

$\begin{array}{llll}\mathrm{C} & .0000000000 & .0000000000 & -.6157446338\end{array}$

$\begin{array}{llll}\mathrm{C} & .0000000000 & .0000000000 & .6157446338\end{array}$

$\mathrm{H} \quad \quad .0000000000 \quad .0000000000-1.6909961542$

$\mathrm{H} \quad \quad .0000000000 \quad .0000000000 \quad 1.6909961542$

Species 8.

$\begin{array}{llrl}\mathrm{C} & .0847511136 & -.6081813828 & .0000000000 \\ \mathrm{C} & .0078439782 & .6881321938 & .0000000000 \\ \mathrm{H} & -.9518340939 & 1.2128772175 & .0000000000 \\ \mathrm{H} & .8763649003 & -1.3575189942 & .0000000000 \\ \mathrm{H} & .9157708030 & 1.3074169258 & .0000000000\end{array}$

Species 9.

$\begin{array}{lrcc}\mathrm{C} & .0234326697 & -.6516062683 & .0000000000 \\ \mathrm{C} & -.0017613636 & .6926287199 & .0000000000 \\ \mathrm{H} & -.9392354988 & 1.2513161939 & .0000000000 \\ \mathrm{H} & .9392752449 & -1.2457721154 & .0000000000 \\ \mathrm{H} & .9444434331 & 1.2379698861 & .0000000000 \\ \mathrm{Cl} & -1.4262682553 & -1.6242199462 & .0000000000\end{array}$


Species 10.

$\begin{array}{lccc}\mathrm{C} & -.6746287338 & .0000000000 & .0000000000 \\ \mathrm{C} & .6746287338 & .0000000000 & .0000000000 \\ \mathrm{H} & -1.2428675304 & -.9338065414 & .0000000000 \\ \mathrm{H} & 1.2428675304 & -.9338065414 & .0000000000 \\ \mathrm{H} & -1.2428675304 & .9338065414 & .0000000000 \\ \mathrm{H} & 1.2428675304 & .9338065414 & .0000000000\end{array}$

Species 11.

$\begin{array}{llll}\mathrm{C} & .2299491133 & -.7005518587 & .0000000000 \\ \mathrm{C} & .0380156093 & .7852743341 & .0000000000 \\ \mathrm{H} & -.5127597199 & 1.1212557611 & -.8923532854 \\ \mathrm{H} & -.5127597199 & 1.1212557611 & .8923532854 \\ \mathrm{H} & .3882727616 & -1.2421598116 & -.9328567695 \\ \mathrm{H} & .3882727616 & -1.2421598116 & .9328567695 \\ \mathrm{H} & 1.0072312811 & 1.3215179087 & .0000000000\end{array}$

Species 12.

$\begin{array}{llcc}\mathrm{C} & .0833286769 & -.7549816999 & .0000000000 \\ \mathrm{C} & .0065692672 & .7639975302 & .0000000000 \\ \mathrm{H} & -.5221063675 & 1.1269771615 & -.8933199252 \\ \mathrm{H} & -.5221063675 & 1.1269771615 & .8933199252 \\ \mathrm{H} & .5861766603 & -1.1424136457 & -.8959910722 \\ \mathrm{H} & .5861766603 & -1.1424136457 & .8959910722 \\ \mathrm{H} & 1.0268677143 & 1.1819491164 & .0000000000 \\ \mathrm{Cl} & -1.5746870640 & -1.4821225680 & .0000000000\end{array}$

Species 13.

$\begin{array}{lrll}\mathrm{C} & .0000000000 & .0000000000 & -.7735157953 \\ \mathrm{C} & .0000000000 & .0000000000 & .7735157953 \\ \mathrm{H} & -.8851255767 & -.5110274900 & 1.1810988783 \\ \mathrm{H} & .8851255767 & -.5110274900 & 1.1810988783 \\ \mathrm{H} & .0000000000 & 1.0220549800 & -1.1810988783 \\ \mathrm{H} & -.8851255767 & -.5110274900 & -1.1810988783 \\ \mathrm{H} & .8851255767 & -.5110274900 & -1.1810988783 \\ \mathrm{H} & .0000000000 & 1.0220549800 & 1.1810988783\end{array}$

Species 14.

$\begin{array}{llll}\mathrm{C} & .0000000000 & .0000000000 & -.7667867698 \\ \mathrm{C} & .0000000000 & .0000000000 & .7667867698 \\ \mathrm{H} & -.8892292780 & -.5133967630 & 1.1646960742 \\ \mathrm{H} & .8892292780 & -.5133967631 & 1.1646960742 \\ \mathrm{H} & .0000000000 & -1.0267935261 & -1.1646960742 \\ \mathrm{H} & .8892292780 & .5133967630 & -1.1646960742 \\ \mathrm{H} & -.8892292780 & .5133967631 & -1.1646960742 \\ \mathrm{H} & .0000000000 & 1.0267935261 & 1.1646960742\end{array}$


Species 15.

$\begin{array}{lllr}\mathrm{C} & .0000000000 & .0000000000 & -.0001745232 \\ \mathrm{H} & -.8670005069 & .0000000000 & .7080162616 \\ \mathrm{H} & .8670005069 & .0000000000 & .7080162616\end{array}$

Species 16.

$\begin{array}{llll}\mathrm{C} & .0000000000 & .0000000000 & .1803312256 \\ \mathrm{H} & -.9965506129 & .0000000000 & .6177633871 \\ \mathrm{H} & .9965506129 & .0000000000 & .6177633871\end{array}$

Species 17.

$\begin{array}{lllc}\mathrm{C} & .0000000000 & .0000000000 & .6798065812 \\ \mathrm{C} & .0000000000 & .0000000000 & -.6388910802 \\ \mathrm{H} & -.9528279485 & .0000000000 & 1.2224097799 \\ \mathrm{H} & .9528279485 & .0000000000 & 1.2224097799\end{array}$

Species 18.

$\begin{array}{llll}\mathrm{C} & -.0584543438 & .0865462986 & .0000000000 \\ \mathrm{H} & .0224754771 & .5901672943 & -.9594679599 \\ \mathrm{H} & .0224754771 & .5901672943 & .9594679599 \\ \mathrm{Cl} & .1135033897 & -1.6210228873 & .0000000000\end{array}$

Species 19.

$\begin{array}{llcc}\mathrm{C} & .0000000000 & .0000000000 & -.0060094728 \\ \mathrm{H} & -.9093162668 & .0000000000 & .6031950588 \\ \mathrm{H} & .9093162668 & .0000000000 & .6031950588 \\ \mathrm{Cl} & .0000000000 & -1.4813390939 & -1.0001903225 \\ \mathrm{Cl} & .0000000000 & 1.4813390939 & -1.0001903225\end{array}$

Species 20.

$\begin{array}{llcl}\mathrm{C} & .0000000000 & .0000000000 & .0000000000 \\ \mathrm{H} & -.5438963133 & .9420560488 & .0000000000 \\ \mathrm{H} & -.5438963134 & -.9420560488 & .0000000000 \\ \mathrm{H} & 1.0877926267 & .0000000000 & .0000000000\end{array}$

Species 21.

$\begin{array}{lccc}\mathrm{C} & .8481115835 & -.1168484493 & .0000000000 \\ \mathrm{C} & -.6306416258 & .0247442595 & .0000000000 \\ \mathrm{H} & 1.0272697395 & -1.2212066372 & .0000000000 \\ \mathrm{H} & -.9966142292 & 1.0594491705 & .0000000000 \\ \mathrm{H} & -1.0388707340 & -.5287166123 & -.8728036218 \\ \mathrm{H} & -1.0388707340 & -.5287166123 & .8728036218\end{array}$

Species 22.

$\begin{array}{llll}\text { C } & .0000000000 & .0000000000 & .0156010179\end{array}$ 


$\begin{array}{lccr}\mathrm{H} & -.5205018485 & .9015356470 & .3586240651 \\ \mathrm{H} & -.5205018485 & -.9015356470 & .3586240651 \\ \mathrm{H} & 1.0410036970 & .0000000000 & .3586240651 \\ \mathrm{Cl} & .0000000000 & .0000000000 & -1.7814643533\end{array}$

Species 23.

$\begin{array}{llll}\mathrm{C} & .0000000000 & .0000000000 & .0000000000 \\ \mathrm{H} & .6340262885 & -.6340262885 & -.6340262885 \\ \mathrm{H} & -.6340262885 & .6340262885 & -.6340262885 \\ \mathrm{H} & -.6340262885 & -.6340262885 & .6340262885 \\ \mathrm{H} & .6340262885 & .6340262885 & .6340262885\end{array}$

Species 24.

$\begin{array}{llll}\mathrm{C} & .4076367118 & .5832342187 & .0000000000 \\ \mathrm{C} & -.2478094943 & -.5266895597 & .0000000000 \\ \mathrm{H} & .8503304341 & 1.5717431183 & .0000000000 \\ \mathrm{H} & 1.0902655917 & -.4326068643 & .0000000000\end{array}$

Species 25.

$\begin{array}{lrrr}\mathrm{Si} & -1.1450884087 & .3427785981 & .0000000000 \\ \mathrm{Si} & 1.1450884087 & -.3427785981 & .0000000000 \\ \mathrm{Cl} & -2.1285763898 & -.4460992560 & 1.6792081056 \\ \mathrm{Cl} & 2.1285763898 & .4460992560 & -1.6792081056 \\ \mathrm{Cl} & -2.1285763898 & -.4460992560 & -1.6792081056 \\ \mathrm{Cl} & 2.1285763898 & .4460992560 & 1.6792081056\end{array}$

Species 26.

\begin{tabular}{|c|c|c|}
\hline $\mathrm{Si}$ & $-.0112845447-1.1635627536$ & .0000000000 \\
\hline $\mathrm{Si}$ & $.0309075216 \quad 1.2018810306$ & .0000000000 \\
\hline $\mathrm{Cl}$ & 1.8491264776 & -1.7102486678 \\
\hline $\mathrm{Cl}$ & $-.9810167396 \quad 1.8491264776$ & 1.7102486678 \\
\hline $\mathrm{Cl}$ & $-1.9554585523-1.8816489508$ & .0000000000 \\
\hline $\mathrm{Cl}$ & $9716510040-1.8517393463$ & -1.6913270618 \\
\hline $\mathrm{Cl}$ & $9716510040-1.8517393463$ & 1.6913270618 \\
\hline
\end{tabular}

Species 27.

$\begin{array}{lccc}\mathrm{Si} & .0000000000 & .0000000000 & -1.1715412374 \\ \mathrm{Si} & .0000000000 & .0000000000 & 1.1715412374 \\ \mathrm{Cl} & -1.6920437717 & -.9769019271 & 1.8546307493 \\ \mathrm{Cl} & 1.6920437717 & -.9769019271 & 1.8546307493 \\ \mathrm{Cl} & .0000000000 & -1.9538038542 & -1.8546307493 \\ \mathrm{Cl} & 1.6920437717 & .9769019271 & -1.8546307493 \\ \mathrm{Cl} & -1.6920437717 & .9769019271 & -1.8546307493 \\ \mathrm{Cl} & .0000000000 & 1.9538038542 & 1.8546307493\end{array}$

Species 28. 


$\begin{array}{lccc}\mathrm{Si} & .0000000000 & .0000000000 & .0005030903 \\ \mathrm{Cl} & -1.6368585383 & .0000000000 & 1.3522619549 \\ \mathrm{Cl} & 1.6368585383 & .0000000000 & 1.3522619549\end{array}$

Species 29.

\begin{tabular}{|c|c|c|c|}
\hline & .0000000000 & .0000000000 & \\
\hline & -.9813121839 & 1.6996825607 & \\
\hline & -.981 & -1.69 & \\
\hline & & .00 & \\
\hline
\end{tabular}

Species 30 .

\begin{tabular}{|c|c|c|}
\hline i & .0000000000 & .0000000000 \\
\hline $\mathrm{Cl}$ & $1.1884292615 \quad 1.1884292615$ & 1.1884292615 \\
\hline & $-1.1884292615-1.1884292615$ & 1.1884292615 \\
\hline $\mathrm{Cl}$ & $615 \quad 1.1884292615$ & -1.188 \\
\hline & $1.1884292615-1.1884292615$ & -1.1884292615 \\
\hline
\end{tabular}

Species 31 .

$\begin{array}{lrrr}\mathrm{Si} & -.2353979634 & .0276992641 & .0000000000 \\ \mathrm{H} & .3846272301 & -.5320451939 & -1.2320687189 \\ \mathrm{H} & .3846272301 & -.5320451939 & 1.2320687189 \\ \mathrm{Cl} & .0661435032 & 2.1085338746 & .0000000000\end{array}$

Species 32 .

\begin{tabular}{|c|c|c|}
\hline & .0000000000 & .0381146203 \\
\hline & -1.2378629013 & .8432103089 \\
\hline $\mathrm{H}$ & 1.2378629013 & .8432103089 \\
\hline$C$ & . $00000000-1.7021717008$. & -1.1622676191 \\
\hline & . $0000000000 \quad 1.7021717008$ & -1.1622676191 \\
\hline
\end{tabular}

Species 33.

$\begin{array}{llll}\mathrm{Si} & .0000000000 & .0000000000 & -.0296548293 \\ \mathrm{H} & -.7055764080 & 1.2220941874 & -.4840921409 \\ \mathrm{H} & -.7055764081 & -1.2220941874 & -.4840921409 \\ \mathrm{H} & 1.4111528161 & .0000000000 & -.4840921409 \\ \mathrm{Cl} & .0000000000 & .0000000000 & 2.0719312520\end{array}$

Species 34.

$\begin{array}{lcccc}\mathrm{Si} & .0502229191 & 1.1253544935 & .0000000000 \\ \mathrm{H} & -1.4738219918 & 1.2458886399 & .0000000000 \\ \mathrm{Cl} & & .0491970728 & -1.0041401334 & .0000000000\end{array}$

Species 35 .

$\begin{array}{lllll}\mathrm{Si} & & .0192004532 & .0673456190 & .0000000000 \\ \mathrm{H} & & -.0135862594 & -1.4213079366 & .0000000000 \\ \mathrm{Cl} & & -.9761274432 & .7381142090 & -1.7116325650\end{array}$ 
$\begin{array}{lllll}\mathrm{Cl} & & -.9761274432 & .7381142090 & 1.7116325650\end{array}$

Species 36.

$\begin{array}{llrr}\mathrm{Si} & .0000000000 & .0000000000 & .0512539529 \\ \mathrm{H} & .0000000000 & .0000000000 & -1.4200581362 \\ \mathrm{Cl} & -.9741634970 & 1.6873006718 & .7457762981 \\ \mathrm{Cl} & -.9741634971 & -1.6873006718 & .7457762981 \\ \mathrm{Cl} & 1.9483269941 & .0000000000 & .7457762981\end{array}$

Species 37.

$\begin{array}{llll}\mathrm{C} & .0000000000 & .0000000000 & 1.8729901588 \\ \mathrm{H} & .0000000000 & -.9443937800 & 2.4220750797 \\ \mathrm{H} & .0000000000 & .9443937800 & 2.4220750797 \\ \mathrm{Si} & .0000000000 & .0000000000 & .1622277834 \\ \mathrm{Cl} & .0000000000 & -1.6845419727 & -1.0181754738 \\ \mathrm{Cl} & .0000000000 & 1.6845419727 & -1.0181754738\end{array}$

Species 38.

$\begin{array}{llll}\mathrm{C} & -.2414229922 & 1.7857276655 & .0000000000 \\ \mathrm{H} & -.3270051271 & 2.3481009184 & -.9330192457 \\ \mathrm{H} & -.3270051271 & 2.3481009184 & .9330192457 \\ \mathrm{Si} & -.0812459701 & -.0455479982 & .0000000000 \\ \mathrm{Cl} & -1.9378023503 & -.9779381976 & .0000000000 \\ \mathrm{Cl} & .9396187680 & -.6753073685 & -1.6901571541 \\ \mathrm{Cl} & .9396187680 & -.6753073685 & 1.6901571541\end{array}$

Species 39.

$\begin{array}{lccc}\mathrm{C} & -.0145844469 & 1.8701589600 & .0000000000 \\ \mathrm{H} & -.9405143767 & 2.4504242446 & .0000000000 \\ \mathrm{H} & .9350136636 & 2.4151990897 & .0000000000 \\ \mathrm{Si} & .0196113555 & .1530433609 & .0000000000 \\ \mathrm{Cl} & -1.6902403941 & -1.0202553085 & .0000000000 \\ \mathrm{H} & 1.2228614886 & -.7001435867 & .0000000000\end{array}$

Species 40.

$\begin{array}{lccc}\mathrm{C} & -.2669675411 & 1.7818262391 & .0000000000 \\ \mathrm{H} & -.3266518976 & 2.3527860628 & -.9302949922 \\ \mathrm{H} & -.3266518976 & 2.3527860628 & .9302949922 \\ \mathrm{Si} & -.1043798301 & -.0586603735 & .0000000000 \\ \mathrm{Cl} & .9448098129 & -.6471554592 & -1.7056733571 \\ \mathrm{Cl} & .9448098129 & -.6471554592 & 1.7056733571 \\ \mathrm{H} & -1.3788831591 & -.8095978936 & .0000000000\end{array}$

Species 41.

$\begin{array}{llll}\mathrm{C} & .0053217776 & 1.8144235922 & .0000000000 \\ \mathrm{H} & .9942928330 & 2.3004317487 & .0000000000\end{array}$ 


$\begin{array}{lccc}\mathrm{H} & -.5629647097 & 2.1497759622 & -.8869569803 \\ \mathrm{H} & -.5629647097 & 2.1497759622 & .8869569803 \\ \mathrm{Si} & .1429015770 & -.0928286016 & .0000000000 \\ \mathrm{Cl} & -1.9564251669 & -.5143583284 & .0000000000\end{array}$

Species 42.

$\begin{array}{llll}\mathrm{C} & -.0071326699 & 1.8558465977 & .0000000000 \\ \mathrm{H} & 1.0393218549 & 2.2033926684 & .0000000000 \\ \mathrm{H} & -.5140541457 & 2.2392838681 & -.8986950593 \\ \mathrm{H} & -.5140541457 & 2.2392838681 & .8986950593 \\ \mathrm{Si} & -.0269071141 & -.0264663110 & .0000000000 \\ \mathrm{Cl} & .9818426097 & -.7194278467 & -1.7047829445 \\ \mathrm{Cl} & .9818426097 & -.7194278467 & 1.7047829445\end{array}$

Species 43.

$\begin{array}{lccc}\mathrm{Si} & .8538088087 & .3259424035 & .4959221889 \\ \mathrm{Cl} & -.9326400521 & .2188387205 & -.6860104414 \\ \mathrm{Cl} & -2.7589957079 & -.6199813604 & .8859521736 \\ \mathrm{Cl} & 1.9855962720 & -1.3542129421 & -.0774979108 \\ \mathrm{C} & 1.6557433218 & 1.9125378336 & -.1767772166 \\ \mathrm{H} & .9827069483 & 2.7656690380 & .0039930197 \\ \mathrm{H} & 2.6000298061 & 2.0733924959 & .3668836528 \\ \mathrm{H} & 1.8612935316 & 1.8193697848 & -1.2541452840\end{array}$

Species 44.

$\begin{array}{llll}\mathrm{C} & .0000000000 & .0000000000 & 1.8568174518 \\ \mathrm{H} & -.5180587849 & .8973041368 & 2.2292002780 \\ \mathrm{H} & -.5180587850 & -.8973041368 & 2.2292002780 \\ \mathrm{H} & 1.0361175699 & .0000000000 & 2.2292002780 \\ \mathrm{Si} & .0000000000 & .0000000000 & .0003135008 \\ \mathrm{Cl} & .9702990300 & -1.6806072186 & -.7359751249 \\ \mathrm{Cl} & .9702990301 & 1.6806072186 & -.7359751249 \\ \mathrm{Cl} & -1.9405980601 & .0000000000 & -.7359751249\end{array}$

Species 45.

$\begin{array}{lccc}\mathrm{C} & .0224209833 & 1.8638595741 & .0000000000 \\ \mathrm{H} & 1.0366314892 & 2.2968132452 & .0000000000 \\ \mathrm{H} & -.5106209302 & 2.2258729157 & -.8928682068 \\ \mathrm{H} & -.5106209302 & 2.2258729157 & .8928682068 \\ \mathrm{Si} & .1137322519 & -.0119726069 & .0000000000 \\ \mathrm{H} & .7822802152 & -.5374054374 & -1.2183924059 \\ \mathrm{H} & .7822802152 & -.5374054374 & 1.2183924059 \\ \mathrm{Cl} & -1.8465807129 & -.7848053536 & .0000000000\end{array}$

Species 46.

$\begin{array}{lllll}\text { C } & .0077290297 & 1.8618881140 & -.0056583875\end{array}$ 


$\begin{array}{lllc}\mathrm{H} & -.5143583260 & 2.2540736571 & -.8934849510 \\ \mathrm{H} & -.5014481756 & 2.2369060252 & .8979730260 \\ \mathrm{H} & 1.0408098314 & 2.2410040987 & -.0075356428 \\ \mathrm{Si} & -.0133154206 & -.0268681160 & -.0195871266 \\ \mathrm{H} & -1.4007435870 & -.5784700517 & .0039312745 \\ \mathrm{Cl} & .9547784134 & -.7298195519 & 1.7190144397\end{array}$

Species 47.

$\begin{array}{lccc}\mathrm{C} & .0030286952 & 1.8608617892 & .0000000000 \\ \mathrm{H} & -.5045514766 & 2.2505254215 & -.8967578399 \\ \mathrm{H} & -.5045514766 & 2.2505254215 & .8967578399 \\ \mathrm{H} & 1.0440504849 & 2.2190724840 & .0000000000 \\ \mathrm{Si} & -.0327524072 & -.0039475350 & .0000000000 \\ \mathrm{Cl} & .9567053301 & -.7191435983 & -1.6951819687 \\ \mathrm{Cl} & .9567053301 & -.7191435983 & 1.6951819687 \\ \mathrm{H} & -1.3886344798 & -.5967447737 & .0000000000\end{array}$

Species 48.

$\begin{array}{llll}\mathrm{C} & .1882284131 & 1.9162478003 & .0000000000 \\ \mathrm{H} & 1.2417671642 & 2.2343115431 & .0000000000 \\ \mathrm{Si} & -.3287257209 & .3290668455 & .0000000000 \\ \mathrm{Cl} & -1.9155421745 & -.9705931937 & .0000000000\end{array}$

Species 49.
$\mathrm{C}$
$\begin{array}{llll}.1499735218 & 1.6477101739 & .5029544657\end{array}$

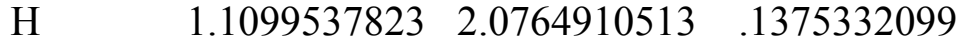
$\begin{array}{llll}\mathrm{Si} & -.1297741212 & .0312278501 & -.2954345180\end{array}$
Cl $\quad \begin{array}{lllll} & -1.9737948417 & -.8476554654 & -.1114667752\end{array}$
$\begin{array}{lllll}\mathrm{Cl} & & .9975136015 & -.0509782983 & 1.5754918941\end{array}$
$\begin{array}{lllll}\mathrm{Cl} & & .8813393265 & -.9779705143 & -1.7890782764\end{array}$

Species 50.

$\begin{array}{lccc}\mathrm{C} & .1728425631 & 1.7428796111 & .0000000000 \\ \mathrm{H} & .8467714091 & 2.5990912324 & .0000000000 \\ \mathrm{Si} & .0469548373 & -.0763934548 & .0000000000 \\ \mathrm{Cl} & -1.9466388073 & -.6192234258 & .0000000000 \\ \mathrm{Cl} & .9576408032 & -.8837649579 & -1.6775348227 \\ \mathrm{Cl} & .9576408032 & -.8837649579 & 1.6775348227\end{array}$




\section{Vibrational frequencies $\left(\mathrm{cm}^{-1}\right)$ at the MP2/aug-cc-pVDZ level.}

Species 1 .

Species 2.

541.2

Species 3.

Species 4.

4427.1

Species 5.

3043.9

Species 6.

$752.1,752.1,2448.2,3568.8$

Species 7.

409.1, 409.1, 703.5, 703.5, 1927.2, 3409.7, 3498.8

Species 8 .

$742.2,961.3,1058.3,1087.5,1416.5,1855.9,3133.8,3259.4,3296.0$

Species 9.

$398.2,628.0,731.9,906.7,960.9,1038.1,1292.5,1404.8,1645.5,3190.5$, $3246.2,3309.0$

Species 10.

833.1, 953.2, 979.4, 1047.8, 1234.5, 1370.5, 1472.9, 1660.6, 3159.5, 3176.5, $3255.1,3283.2$

Species 11.

144.1, 469.7, 809.0, 977.3, 1088.6, 1193.8, 1389.0, 1466.4, 1475.0, 1494.0, $3011.9,3101.8,3151.4,3194.0,3310.6$

Species 12 .

258.8, 338.1, 688.6, 790.0, 995.0, 1082.4, 1102.1, 1269.8, 1311.1, 1397.9, $1474.3,1484.6,1490.8,3056.3,3106.6,3149.5,3163.4,3185.0$

Species 13.

$314.5,900.5$, 900.8, 1020.3, 1162.6, 1164.4, 1381.2, 1435.4, 1490.2, 1490.4, $1500.1,1500.6,3054.6,3062.9,3133.2,3134.3,3154.9,3155.9$

Species 14.

321.0, 822.0, 822.3, 1023.4, 1214.1, 1215.3, 1387.7, 1415.3, 1495.4, 1495.8, 
1496.2, 1496.4, 3049.9, 3051.3, 3129.6, 3130.6, 3150.4, 3151.2

Species 15.

$1414.0,2961.7,3043.0$

Species 16.

$1146.9,3165.0,3403.0$

Species 17.

295.5, 734.9, 1217.9, 1670.0, 3142.7, 3251.8

Species 18 .

301.7, 852.6, 1018.3, 1439.3, 3204.8, 3367.6

Species 19.

288.9, 725.2, 776.4, 918.3, 1181.1, 1286.2, 1457.1, 3133.3, 3224.7

Species 20.

$494.6,1424.5,1425.7,3141.4,3341.8,3344.2$

Species 21 .

$450.5,810.1,888.1,1083.1,1240.5,1288.0,1448.8,1450.1,2978.0,3005.1$, $3052.5,3159.8$

Species 22 .

747.1, 1035.8, 1036.2, 1371.6, 1473.3, 1474.0, 3084.9, 3205.4, 3207.5

Species 23.

1329.8, 1329.8, 1329.8, 1555.4, 1555.4, 3052.4, 3196.8, 3196.8, 3196.8

Species 24.

536.5, 649.7, 1099.4, 1749.8, 2499.4, 3370.9

Species 25 .

$39.8,52.7,95.5,116.0,136.2,183.0,215.8,296.7,485.1,534.6$, $552.3,560.1$

Species 26.

$24.5,54.8,64.9,118.1,128.4,167.6,188.0,193.3,234.3,356.1$, $476.3,565.7,580.8,591.8,596.2$

Species 27.

$24.1,57.0,57.4,118.3,118.5,125.1,167.1,167.5,202.6,203.0$, $233.0,346.0,453.4,585.4,586.6,602.9,604.0,624.8$

Species 28. 
$189.2,495.4,505.5$

Species 29.

$157.8,158.0,232.9,464.2,576.0,577.8$

Species 30 .

138.4, 139.1, 211.4, 211.4, 211.6, 412.3, 609.9, 609.9, 610.1

Species 31 .

$531.4,648.9,745.8,928.0,2232.3,2270.9$

Species 32 .

$180.7,515.1,575.9,602.3,710.3,872.8,956.7,2304.4,2327.8$

Species 33.

534.0, 661.2, 661.3, 952.0, 964.5, 964.8, 2277.2, 2294.4, 2294.8

Species 34 .

$502.5,815.3,2087.7$

Species 35 .

$173.2,504.8,565.4,664.4,747.1,2255.9$

Species 36 .

$166.9,167.1,242.5,483.7,590.5,592.3,794.8,795.4,2362.2$

Species 37.

171.6, 185.0, 210.2, 478.6, 535.9, 597.7, 603.8, 757.2, 1010.6, 1362.6, $3168.4,3292.8$

Species 38 .

$45.8,145.0,158.4,215.1,218.2,228.2,438.4,541.0,575.8,601.2$,

$786.2,811.4,1404.0,3178.2,3304.1$

Species 39 .

213.7, 333.5, 532.4, 591.5, 667.6, 715.8, 860.4, 986.2, 1374.8, 2336.1, $3168.8,3292.5$

Species 40.

43.3, 168.9, 196.4, 255.7, 486.4, 560.5, 574.3, 731.2, 767.7, 815.0, $838.8,1405.8,2311.8,3176.4,3297.9$

Species 41 .

$75.8,232.2,481.3,569.9,653.2,775.6,1239.3,1410.5,1425.2,3023.3$, $3122.1,3146.1$ 
Species 42.

$155.9,165.3,184.4,234.0,473.5,547.2,712.7,790.4,815.7,1257.2$, $1429.6,1436.4,3047.1,3155.1,3173.4$

Species 43.

$40.2,86.1,158.2,168.5,195.6,247.0,292.3,512.2,566.4,689.6$, 814.6, 929.8, 1252.4, 1432.0, 1441.6, 3061.3, 3163.9, 3188.0

Species 44.

$149.2,149.6,197.6,212.6,213.0,224.7,441.2,573.0,574.7,764.8$, $830.8,831.7,1283.7,1438.2,1438.8,3050.1,3162.4,3164.3$

Species 45.

$159.3,192.2,494.2,513.2,683.8,700.1,756.7,892.8,914.5,971.1$, $1279.9,1440.5,1445.8,2267.8,2278.5,3051.3,3152.3,3168.2$

Species 46.

$129.5,189.0,508.2,550.3,679.8,727.8,807.2,888.3,1265.0,1433.5$, $1438.6,2241.5,3053.6,3155.3,3173.4$

Species 47.

$160.8,171.1,193.5,239.1,488.9,557.4,680.6,755.8,757.8,855.5$, $897.9,1280.4,1438.2,1440.1,2318.6,3052.4,3162.1,3163.5$

Species 48.

246.7, 536.0, 607.5, 645.9, 1150.0, 3188.4

Species 49.

$116.2,151.2,192.5,216.4,220.3,435.9,480.6,595.5,744.9,867.8$, $966.1,3060.3$

Species 50.

$116.3,156.0,181.2,227.7,235.5,256.1,450.5,523.8,585.5,610.0$, $841.2,3287.5$ 
D. Isobaric heat capacities (cal/(mol K)) at atmospheric pressure at the MP2/aug-ccpVDZ level.

\begin{tabular}{|c|c|c|c|c|c|c|c|c|c|c|}
\hline \multirow{2}{*}{ Species } & \multicolumn{10}{|c|}{ Temperatures (Kelvin degrees) } \\
\hline & 298.15 & 400 & 600 & 800 & 1000 & 1200 & 1400 & 1600 & 1800 & 2000 \\
\hline 1. $\mathrm{Cl}$ & 5.0 & 5.0 & 5.0 & 5.0 & 5.0 & 5.0 & 5.0 & 5.0 & 5.0 & 5.0 \\
\hline 2. $\mathrm{Cl}_{2}$ & 8.1 & 8.4 & 8.7 & 8.8 & 8.8 & 8.9 & 8.9 & 8.9 & 8.9 & 8.9 \\
\hline 3. $\mathrm{H}$ & 5.0 & 5.0 & 5.0 & 5.0 & 5.0 & 5.0 & 5.0 & 5.0 & 5.0 & 5.0 \\
\hline 4. $\mathrm{H}_{2}$ & 7.0 & 7.0 & 7.0 & 7.0 & 7.1 & 7.2 & 7.4 & 7.6 & 7.7 & 7.9 \\
\hline 5. $\mathrm{HCl}$ & 7.0 & 7.0 & 7.0 & 7.2 & 7.4 & 7.7 & 7.9 & 8.1 & 8.2 & 8.3 \\
\hline 6. $\mathrm{C}_{2} \mathrm{H}$ & 8.4 & 9.2 & 10.2 & 11.0 & 11.6 & 12.2 & 12.7 & 13.0 & 13.3 & 13.6 \\
\hline 7. $\mathrm{C}_{2} \mathrm{H}_{2}$ & 11.5 & 12.8 & 14.3 & 15.3 & 16.2 & 17.0 & 17.6 & 18.2 & 18.6 & 18.9 \\
\hline 8. $\mathrm{C}_{2} \mathrm{H}_{3}$ & 9.9 & 11.6 & 14.6 & 16.8 & 18.5 & 19.9 & 21.0 & 21.8 & 22.5 & 23.0 \\
\hline 9. $\mathrm{C}_{2} \mathrm{H}_{3} \mathrm{Cl}$ & 12.7 & 15.4 & 19.4 & 22.1 & 24.1 & 25.6 & 26.7 & 27.6 & 28.3 & 28.8 \\
\hline 10. $\mathrm{C}_{2} \mathrm{H}_{4}$ & 10.1 & 12.5 & 16.6 & 19.7 & 22.1 & 23.9 & 25.4 & 26.5 & 27.4 & 28.0 \\
\hline 11. $\mathrm{C}_{2} \mathrm{H}_{5}$ & 13.1 & 15.6 & 20.1 & 23.6 & 26.3 & 28.5 & 30.2 & 31.5 & 32.5 & 33.3 \\
\hline 12. $\mathrm{C}_{2} \mathrm{H}_{5} \mathrm{C}$ & 14.4 & 17.9 & 23.7 & 28.0 & 31.3 & 33.7 & 35.6 & 37.0 & 38.2 & 39.0 \\
\hline 13. $\mathrm{C}_{2} \mathrm{H}_{6} \mathrm{C}$ & 10.3 & 13.3 & 19.1 & 23.7 & 27.3 & 30.1 & 32.3 & 34.0 & 35.3 & 36.3 \\
\hline 14. $\mathrm{C}_{2} \mathrm{H}$ & 12.1 & 15.2 & 21.1 & 25.7 & 29.3 & 32.1 & 34.3 & 36.0 & 37.3 & 38.3 \\
\hline 15. ${ }^{1} \mathrm{CH}_{2}$ & 8.1 & 8.3 & 8.9 & 9.7 & 10.4 & 11.0 & 11.5 & 11.9 & 12.2 & 12.5 \\
\hline $16 .{ }^{3} \mathrm{CH}_{2}$ & 8.2 & 8.5 & 9.1 & 9.7 & 10.3 & 10.9 & 11.4 & 11.8 & 12.1 & 12.4 \\
\hline 17. $\mathrm{CH}_{2} \mathrm{C}$ & 10.6 & 11.6 & 13.1 & 14.4 & 15.4 & 16.2 & 16.9 & 17.4 & 17.8 & 18.1 \\
\hline 18. $\mathrm{CH}_{2} \mathrm{Cl}$ & 10.6 & 11.7 & 13.4 & 14.6 & 15.5 & 16.3 & 16.9 & 17.4 & 17.8 & 18.1 \\
\hline 19. $\mathrm{CH}$ & 12.1 & 14.1 & 17.2 & 19.2 & 20.6 & 21.6 & 22.4 & 23.0 & 23.5 & 23.9 \\
\hline 20. $\mathrm{CH}_{3}$ & 9.4 & 10.1 & 11.5 & 12. & 13.9 & 14.9 & 15.7 & 16.4 & 16.9 & 17.4 \\
\hline 21. $\mathrm{CH}_{3} \mathrm{C}$ & 9.9 & 12.0 & 15.8 & 18. & 20.9 & 22.6 & 24.0 & 25.0 & 25.8 & 26.4 \\
\hline 22. $\mathrm{CH}_{3} \mathrm{C}$ & 9.6 & 11.4 & 14.5 & 16.8 & 18.6 & 20.0 & 21.1 & 21.9 & 22.6 & 23.1 \\
\hline 23. $\mathrm{CH}_{4}$ & 8.5 & 9.6 & 12.3 & 14.8 & 16.8 & 18.5 & 19.8 & 20.9 & 21.7 & 22.3 \\
\hline 24. $\mathrm{HCHC}$ & 10.4 & 11.5 & 13.2 & 14.6 & 15.6 & 16.5 & 17.1 & 17.6 & 18.0 & 18.3 \\
\hline 25. $\mathrm{Si}_{2} \mathrm{Cl}_{4}$ & 27.8 & 29.3 & 30.6 & 31.1 & 31.3 & 31.5 & 31.6 & 31.6 & 31.7 & 31.7 \\
\hline 26. $\mathrm{Si}_{2} \mathrm{C}$ & 2.2 & 34.3 & 36.1 & 36.8 & 37.1 & 37.3 & 37.4 & 37.5 & 37.6 & 37.6 \\
\hline 27. $\mathrm{Si}_{2} \mathrm{Cl}_{6}$ & 37.0 & 39.5 & 41.6 & 42.5 & 42.9 & 43.2 & 43.3 & 43.4 & 43.5 & 43.5 \\
\hline $28 . s$ & 12.3 & 12.9 & 13.4 & 13.6 & 13.7 & 13.8 & 13.8 & 13.8 & 13.9 & 13.9 \\
\hline 29. $\mathrm{SiC}$ & 17.0 & 18.1 & 19.0 & 19.4 & 19.5 & 19.6 & 19.7 & 19.7 & 19.8 & 19.8 \\
\hline 30. $\mathrm{SiCl}_{4}$ & 21.7 & 23.2 & 24.6 & 25.1 & 25.3 & 25.5 & 25.6 & 25.6 & 25.7 & 25.7 \\
\hline 31. $\mathrm{SiH}_{2} \mathrm{Cl}$ & 11.3 & 12.8 & 14.7 & 16.0 & 17.0 & 17.7 & 18.1 & 18.5 & 18.7 & 18.9 \\
\hline 32. $\mathrm{SiH}_{2} \mathrm{Cl}_{2}$ & 14.9 & 17.1 & 19.8 & 21.4 & 22.5 & 23.3 & 23.9 & 24.2 & 24.5 & 24.8 \\
\hline 33. $\mathrm{SiH}_{3} \mathrm{Cl}$ & 12.2 & 14.5 & 17.6 & 19.7 & 21.2 & 22.3 & 23.0 & 23.6 & 24.0 & 24.3 \\
\hline 34. $\mathrm{SiHCl}$ & 9.8 & 10.6 & 11.5 & 12. & 12.6 & 12.9 & 13.1 & 13.3 & 13.4 & 13.5 \\
\hline 35. $\mathrm{SiHCl}$ & 13.8 & 15.2 & 16.8 & 17.7 & 18.2 & 18.6 & 18.9 & 19.1 & 19.2 & 19.4 \\
\hline 36. $\mathrm{SiHC}$ & 18.2 & 20.1 & 22.1 & 23.2 & 23.9 & 24.4 & 24.7 & 24.9 & 25.1 & 25.2 \\
\hline 37. $\mathrm{CH}$ & 19.2 & 21.7 & 24.4 & 26.0 & 27.2 & 28.1 & 28.7 & 29.3 & 29.7 & 30.0 \\
\hline 38. $\mathrm{CH}_{2} \mathrm{Si}$ & 25.2 & 27.7 & 30.5 & 32.1 & 33.2 & 34.0 & 34.7 & 35.2 & 35.6 & 36.0 \\
\hline 39. $\mathrm{CH}_{2} \mathrm{~s}$ & 16.4 & 19.0 & 22.3 & 24.3 & 25.9 & 27.0 & 27.9 & 28.6 & 29.1 & 29.5 \\
\hline 40. $\mathrm{CH}_{2} \mathrm{SiHCl}_{2}$ & 21.7 & 24.6 & 28.1 & 30.2 & 31.8 & 33.0 & 33.9 & 34.5 & 35.1 & 35.5 \\
\hline
\end{tabular}




\begin{tabular}{|l|l|l|l|l|l|l|l|l|l|l|}
\hline 41. $\mathrm{CH}_{3} \mathrm{SiCl}$ & 16.1 & 18.2 & 21.3 & 23.5 & 25.1 & 26.4 & 27.4 & 28.2 & 28.8 & 29.2 \\
\hline 42. $\mathrm{CH}_{3} \mathrm{SiCl}_{2}$ & 20.3 & 23.0 & 26.6 & 29.0 & 30.8 & 32.1 & 33.2 & 34.0 & 34.6 & 35.1 \\
\hline $43 . \mathrm{CH}_{3} \mathrm{SiCl}_{2} \mathrm{Cl}$ & 25.6 & 28.4 & 32.2 & 34.8 & 36.6 & 38.0 & 39.0 & 39.9 & 40.5 & 41.0 \\
\hline $44 . \mathrm{CH}_{3} \mathrm{SiCl}_{3}$ & 24.9 & 28.0 & 32.0 & 34.6 & 36.5 & 37.9 & 39.0 & 39.8 & 40.5 & 41.0 \\
\hline 45. $\mathrm{CH}_{3} \mathrm{SiH}_{2} \mathrm{Cl}$ & 18.3 & 22.0 & 27.3 & 31.0 & 33.8 & 35.8 & 37.3 & 38.5 & 39.4 & 40.1 \\
\hline 46. $\mathrm{CH}_{3} \mathrm{SiHCl}$ & 17.3 & 20.2 & 24.4 & 27.3 & 29.5 & 31.1 & 32.4 & 33.3 & 34.1 & 34.7 \\
\hline 47. $\mathrm{CH}_{3} \mathrm{SiHCl}_{2}$ & 21.5 & 24.9 & 29.7 & 32.8 & 35.1 & 36.8 & 38.2 & 39.2 & 39.9 & 40.5 \\
\hline 48. $\mathrm{CHSiCl}^{1}$ & 13.1 & 14.5 & 16.1 & 16.9 & 17.5 & 18.0 & 18.3 & 18.6 & 18.8 & 19.0 \\
\hline 49. ${ }^{1} \mathrm{CHSiCl}_{3}$ & 22.7 & 24.9 & 27.3 & 28.5 & 29.3 & 29.8 & 30.2 & 30.5 & 30.7 & 30.9 \\
\hline $50 .{ }^{3} \mathrm{CHSiCl}_{3}$ & 24.2 & 26.2 & 28.0 & 28.9 & 29.5 & 29.9 & 30.3 & 30.5 & 30.7 & 30.9 \\
\hline
\end{tabular}

